\title{
Transradial access chemoembolization for hepatocellular carcinoma in comparation with transfemoral access
}

\author{
Nan Du ${ }^{1,2}$, Min-Jie Yang ${ }^{1,2}$, Jing-Qin $M^{1,2}$, Jian-Jun Luo ${ }^{1,2}$, Zi-Han Zhang ${ }^{1}$, Tian-Zhu Yu ${ }^{1}$, Zhi-Yuan Zheng ${ }^{1,2}$, \\ Wen Zhang ${ }^{1,2}$, Zhi-Ping Yan ${ }^{1,2}$ \\ ${ }^{1}$ Department of Interventional Radiology, Zhongshan Hospital, Fudan University, Shanghai 200032, China; ${ }^{2}$ Shanghai Institute of Medical Imaging, \\ Shanghai 200041, China \\ Contributions: (I) Conception and design: ZP Yan, W Zhang; (II) Administrative support: W Zhang, JJ Luo; (III) Provision of study materials or \\ patients: JQ Ma, ZH Zhang, W Zhang, ZP Yan; (IV) Collection and assembly of data: N Du, MJ Yang, TZ Yu; (V) Data analysis and interpretation: \\ N Du, MJ Yang, ZY Zheng; (VI) Manuscript writing: All authors; (VII) Final approval of manuscript: All authors. \\ Correspondence to: Wen Zhang. Department of Interventional Radiology, Zhongshan Hospital, Fudan University, No. 180 Fenglin Road, Xuhui \\ District, Shanghai 200032, China. Email: Zhangwen_zs@126.com; Zhi-Ping Yan. Department of Interventional Radiology, Zhongshan Hospital, \\ Fudan University, No. 180 Fenglin Road, Xuhui District, Shanghai 200032, China. Email: Yanzhiping@126.com.
}

\begin{abstract}
Background: This study aimed to compare the safety and efficacy of transradial access (TRA) with transfemoral access (TFA) chemoembolization in treatment of hepatocellular carcinoma (HCC).

Methods: HCC patients who were late for curative treatment on initial diagnosis or HCC patients who had undergone one or several rounds of transarterial chemoembolization (TACE) were enrolled. The clinical and angiographic characteristics, the procedure related details, and the follow-up data from patients who underwent TRA and TFA were analyzed and compared.

Results: In total, 112 patients undergoing 160 TRA-TACE and 107 patients undergoing 163 TFA-TACE were included. The technical success rate of TRA was $95.0 \%$ and that of TFA was $98.8 \%(\mathrm{P}=0.102)$. In the TFA-TACE group, $5.5 \%$ of cases suffered access site-related complications, including 6 with minor bleeding and 3 with severe bleeding or pseudoaneurysm. In the TRA-TACE group, 1.9\% of cases underwent crossover to femoral access for selective cannulation failure. The rate of radial artery occlusion (RAO) was $2.7 \%$ (3 of 112 patients), and none of the RAO patients suffered paresthesia, pain at the site of occlusion, hand function loss or distal ischemia. Comparing patients with/without access site-related complications in the TFA-TACE group, there was a statistical difference in patient age and in the percentage of patient with a PT time $>15$ s $(72.6 \%$ vs. $57.1 \%, \mathrm{P}<0.001 ; 44.4 \%$ vs. $11.7 \%, \mathrm{P}=0.022)$.
\end{abstract}

Conclusions: TRA is a safe and effective method for patients undergoing TACE. Compared with TFA, TRA may reduce the occurrence of access site-related bleeding and vascular complications. TRA-TACE may especially benefit older patients or those with a longer prothrombin time (PT).

Keywords: Transarterial chemoembolization (TACE); transradial access (TRA); transfemoral access (TFA); access site-related complication.

Submitted Feb 21, 2019. Accepted for publication Aug 12, 2019.

doi: $10.21037 /$ tcr.2019.08.40

View this article at: http://dx.doi.org/10.21037/tcr.2019.08.40

\section{Introduction}

Transarterial chemoembolization (TACE) is an effective treatment procedure for patients with unresectable hepatocellular carcinoma (HCC), which was often performed via femoral artery (FA) access (1). In recent years, transradial access (TRA) has emerged as a new approach, and it is commonly used in coronary angioplasty, as well as in stent placement (2,3). Compared with transfemoral access (TFA), transradial percutaneous coronary intervention (PCI) has a variety of advantages, including overall increased 
procedure comfort, decreased procedure access site-related major bleeding and better outcomes $(3,4)$. For unresectable HCC, repetitive TACE has been recognized as a common and effective treatment approach $(5,6)$. Several studies compared the safety and efficacy of TRA with that of TFA for patients undergoing TACE treatment $(1,7,8)$. In these studies, TRA demonstrated its superiority over TFA in a series of indexes, including the monitoring time after procedure, time of ambulation and hospital stay, the rate of bleeding risk, and fluoroscopy time. However, shortcomings of TRA also exist, such as puncture failure, challenging cannulation and the possible occlusion of the radial artery (RA) $(9,10)$. Moreover, potential increased radiation exposure, increased distance from the access site and a long learning curve also remain as potential barriers preventing TRA usage by an interventional radiologist (IR) $(11,12)$.

A previous study indicated that radial artery occlusion (RAO) occurred in $13.7 \%$ cases with 5 -Fr sheath $(\mathrm{n}=153)$ and in $30.5 \%$ cases with $6-\mathrm{Fr}$ sheath $(\mathrm{n}=302)$ after TRA coronary catheterization (13). RAO is generally asymptomatic due to dual blood supply to hands; however, it can affect the future use of the RA for hemodialysis fistula preparation (14), coronary artery bypass grafting (15), reconstructive surgery (16). Most importantly, it may prevent the ability to repeat the TRA. Whether repeated usage of radial puncture for TACE is clinically feasible or not, it remains unconfirmed. Also, a comparative study is needed to determine the safety and efficacy of TRA. Hence, the aim of this study was to investigate the safety and efficacy of TRA in patients undergoing TACE treatments compared with TFA and to evaluate the risk factors of access site-related bleeding complications of TFA.

\section{Methods}

This single-center retrospective study was approved by the local Institutional Review Board. HCC patients who were not suitable for curative treatment and had undergone TACE in Zhongshan Hospital, Fudan University from Oct 2017 to Oct 2018, were enrolled. All patients were rated as intermediate stage (B), according to the Barcelona Clinic Liver Cancer (BCLC) staging classification, and as level A or B according to the Child-Pugh grading system. All the TACE procedures were monitored by a chief IR with over 20 years of clinical experience and were operated by his professional medical team. Allen testing was done prior to each TRA-TACE to confirm dual circulation of the hand and exclude the contraindicated patients. Other exclusion criteria included a RA diameter $<2 \mathrm{~mm}$ via ultrasound, a previous history of severe vascular tortuosity or RAO, severe peripheral vascular disease, and patients who needed dialysis. The access route decision was determined by the patient and IR's joint consultation after a comprehensive evaluation, and the access route was usually unchanged in a subsequent TACE treatment; otherwise the occurrence of access site exchange was recorded and analyzed. In total, 219 patients who had undergone 323 times of TACE procedures were enrolled in this study. Patients were divided into two groups based on different TACE access site: TRATACE group or TFA-TACE group. Patient demographics and clinical data included age, sex, height, body mass index (BMI), hypertension, diabetic mellitus, previous TACE, anticoagulant therapy, history of hepatitis, international normalized ratio (INR), prothrombin time (PT), ChildPugh classification, and tumor burdens. Five clinical and laboratory measures (total bilirubin, serum albumin, prothrombin ratio, ascites, and hepatic encephalopathy) were also recorded to calculate the Child-Pugh score of patients (17). The angiographic characteristics of patients were recorded including tumor burden and tumor location. Clinical outcomes evaluated included technical success, access site-related bleeding and vascular complications, the level of comfort, neurologic complications within 30 days and contrast medium-induced nephropathy.

Technical success was defined as successful RA puncture and catheterization of target artery. Failure to puncture or catheterize the target vessel was defined as a technical failure. According to the quality improvement guidelines from the Society of Interventional Radiology (SIR) (18), access site-related complications were categorized in as major and minor. Major complications were those that needed prolonged hospitalization, an unplanned increase in the level of care, permanent adverse sequelae, and death. Such complications included severe bleeding, obvious hematomas, arterial dissection, arterial pseudoaneurysm, arteriovenous fistula and symptomatic vascular occlusion that required an intervention such as thrombin injection, transfusion, angioplasty, surgical repair or ultrasoundguided compression. Minor complications included those that needed additional nominal therapy and overnight admission for observation, such as asymptomatic RAO, hematoma or blood loss not requiring transfusion or open surgical repair. Also, patients with postoperative dysuria requiring urethral catheterization were also recorded as a factor reflecting the comfort of patient. Neurologic events after the procedure were recorded, including transient 

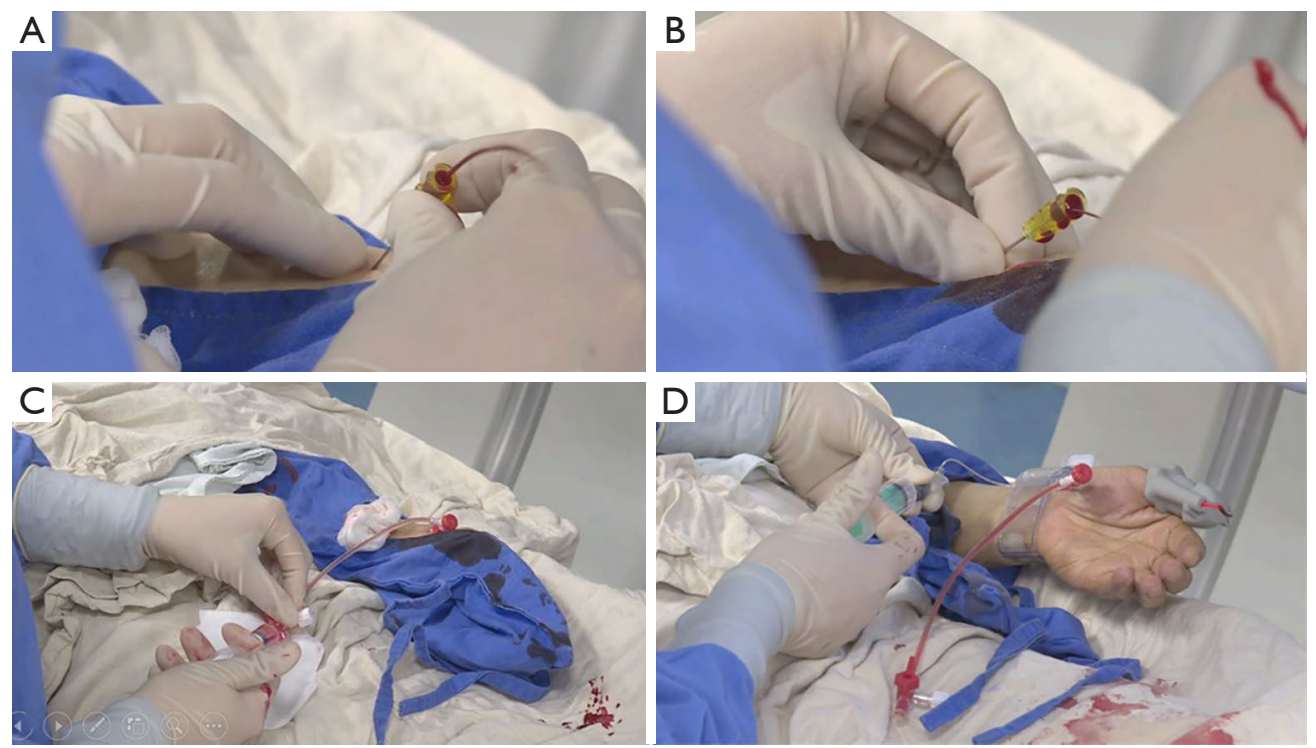

Figure 1 Transradial puncture and hemostasis by a special Band. (A) Blood spills through the puncture needle after radial artery was successful punctured with a 20-gauge needle (Merit Medical, South Jordan, Utah, USA); (B) a 0.025-inch guidewire was inserted into radial artery; (C) a cocktail solution including heparin $30 \mathrm{mg}$, nitroglycerin $0.1 \mathrm{mg}$, and $20 \mathrm{mg}$ lidocaine was given to prevent radial artery spasm after a 4-Fr sheath insertion into radial artery; (D) a special Band device was used for hemostasis after transarterial chemoembolization (TACE) procedure.

ischemic attacks, reversible ischemic neurologic deficits and stroke, which was defined as a new, persistent neurologic disability lasting more than 24 hours. Contrast-induced nephropathy was defined as either an increase in the serum creatinine level of more than $25 \%$ or an absolute increase in the serum creatinine level of $0.5 \mathrm{mg} / \mathrm{dL}$.

\section{Procedures and follow-up}

All patients signed informed consent before operation. For patients with a moderate/high preoperative bleeding tendency, preoperative bleeding prevention strategies were performed, including anticoagulant discontinuance before operation, vitamin $\mathrm{K}$ injection and/or liver function protection (19). A pre-procedural RA assessment was performed, including the Allen test, as well as an inner diameter inspection by ultrasound. The left RA puncture was performed one centimeter proximal to the styloid process of the radius under local anesthesia (Figure 1A,B). After puncture success, a 4-Fr or 5-Fr Sheath Introducer (Merit Medical, South Jordan, Utah, USA) was exchanged, utilizing the Seldinger technique. After the sheath was introduced, a cocktail solution containing heparin $20-30 \mathrm{mg}$, nitroglycerin $0.1 \mathrm{mg}$ and lidocaine $20 \mathrm{mg}$ was given through the sheath to prevent RA spasm (Figure 1C). Heparin was decreased or not administered in patients with moderate or high bleeding risk (INR $\geq 1.5$ ), as regulated by the consensus guideline of SIR and Cardiovascular and Interventional Radiology Society of Europe (SIR/CIRSE) (19). Then, a $125 \mathrm{~cm}, 4-\mathrm{Fr}$ MPA catheter was inserted through the sheath to the aorta over a hydrophilic guide wire. The catheter was used to cannulate the celiac trunk, hepatic artery, superior mesenteric arteries or inferior phrenic artery, if needed. After finding the vessels responsible for supplying the tumor, a 2.7-Fr microcatheter (Terumo Corp, Tokyo, Japan) was introduced over a hydrophilic guide wire. Digital subtraction angiography was performed to identify the tumor vascularity and stain prior to the embolization.

The TACE procedures performed did not included drug-eluting bead embolization or radio-embolization by yttrium 90 microspheres. After the procedure, the vascular sheath was removed immediately, and hemostasis was performed with a special Band device (Terumo Corp) (Figure 1D). As the band was deflated incrementally and removed six hours after operation, arterial hemostasis and pulse were reconfirmed. Also, prior to discharge and during a follow-up at about 30 days after discharge, repeat evaluations of the access site and radial pulse were 
conducted. RAO is defined as the absence of a RA pulse after a negative reversed Allen test, or is defined by a visible obstruction observed by ultrasound.

Transfemoral artery puncture was performed through the right common FA. Then, an $80 \mathrm{~cm}$, 4-Fr or 5 -Fr RH catheter was introduced to the aorta. A microcatheter/microwire was inserted into the responsible vessel, subsequently. Embolization was performed using the same technique as described for the TRA-TACE. After the procedure was done, the femoral sheath was removed immediately followed by compression homeostasis and a pressure bandage wrap. The patients were required to follow bed rest while keeping the lower limbs extended at for least six hours before the hemostatic bandage could be removed. Access site-related complications were evaluated and recorded before discharge.

All patients were closely followed up after TACE. In both groups, if new tumor nodules were evident on CT scans and if the initial lesions seemed to revascularize, then a subsequent TACE treatment was applied. A comprehensive evaluation by IR was necessary to decide the timing of the treatment, as well as the access route, before each therapy.

\section{Statistical analysis}

Statistical analysis was performed using SPSS 22.0 (SPSS, Chicago, IL, USA). Categorical variables were presented as absolute number with percentage, and continuous variables were presented as mean \pm standard deviation. The differences between the groups were evaluated by using the Chi-square test or Fisher exact test for categorical variables and the 2-tailed Student $t$ test for continuous variables. A two-side $\mathrm{P}$ value $<0.05$ was statistical significance.

\section{Results}

From October 2017 to October 2018, 219 patients undergoing a total of 323 times TACE procedures were included in this study. Of those, 112 patients underwent 160 TRA-TACE procedures, while 107 patients underwent 163 TFA-TACE procedures. The baseline clinical characteristics of patients are presented in Table 1. No significant difference was found between the two groups regarding baseline data, such as age, sex, height, BMI, comorbidities and liver function stage. The number of patients receiving TRATACE for the first time was significantly higher than that via $\mathrm{FA}$ access $(37.5 \%$ vs. $15.9 \%, \mathrm{P}<0.001)$. No substantial difference in the number of TACE procedures or the mean of TACE treatments per patient were found between the
TRA-TACE group and the TFA-TACE group.

The clinical data and outcomes of each procedure for the two groups are recorded in Table 2. Overall technical success was achieved in $95.0 \%$ of cases in the TRA-TACE group and $98.8 \%$ of cases in the TFA-TACE group $(\mathrm{P}=0.102)$. TRA had a lower rate of access site-related complications compared to TFA, although the differences in the rates was not statistically significant $(1.9 \%$ vs. $5.5 \%, \mathrm{P}=0.083)$. Three cases $(1.9 \%)$ with asymptomatic RAO were noted in the TRA-TACE group, while in the TFA-TACE group, six cases $(3.7 \%)$ were observed with minor complication, such as ecchymosis in the inguinal region. Additionally, no major complications occurred in the TRA-TACE group, while three cases $(1.8 \%)$ had major complications in the TFATACE group, including obvious access site bleeding in two cases that received thrombin injection or blood transfusion and a pseudoaneurysm in one case that received ultrasonic guided compression. Five cases $(3.1 \%)$ had postoperative dysuria that needed urethral catheterization in the TFATACE group. No neurologic complications or contrast medium-induced nephropathy were founded in any cases during follow-up.

In the TFA-TACE group, the characteristics of patients with/without access site-related bleeding complications were compared and presented in Table 3. Patients with advanced age were more likely to suffer access siterelated complications $(\mathrm{P}<0.001)$. Also, patients with $\mathrm{PT}$ $>15 \mathrm{~s}$ were more prone to suffering access site-related complications $(\mathrm{P}=0.022)$.

\section{Discussion}

TRA interventional therapy has significantly grown worldwide in recent years, especially in cardiac interventions. Despite established data that has demonstrated transradial PCI with lower rates of access site-related bleeding complications compared to transfemoral PCI, this technique is rarely used by IRs in the systemic circulation. In the present study, we compared the clinical and angiographic characteristics, procedure details, and related complications of TRA-TACE with those of TFA-TACE in order to evaluate the efficacy and safety of TRA-TACE. We also analyzed the predictors of access site-related bleeding complications in TFA-TACE group. Our results indicate that, firstly, compared with TFA, TRA is associated with significantly lower rates of access site-related bleeding complications without compromising procedural success rates. Second, TFA patients with advanced age or PT $>15 \mathrm{~s}$ are more 
Table 1 Demographic and clinical data of patients in TRA-TACE group and TFA-TACE group

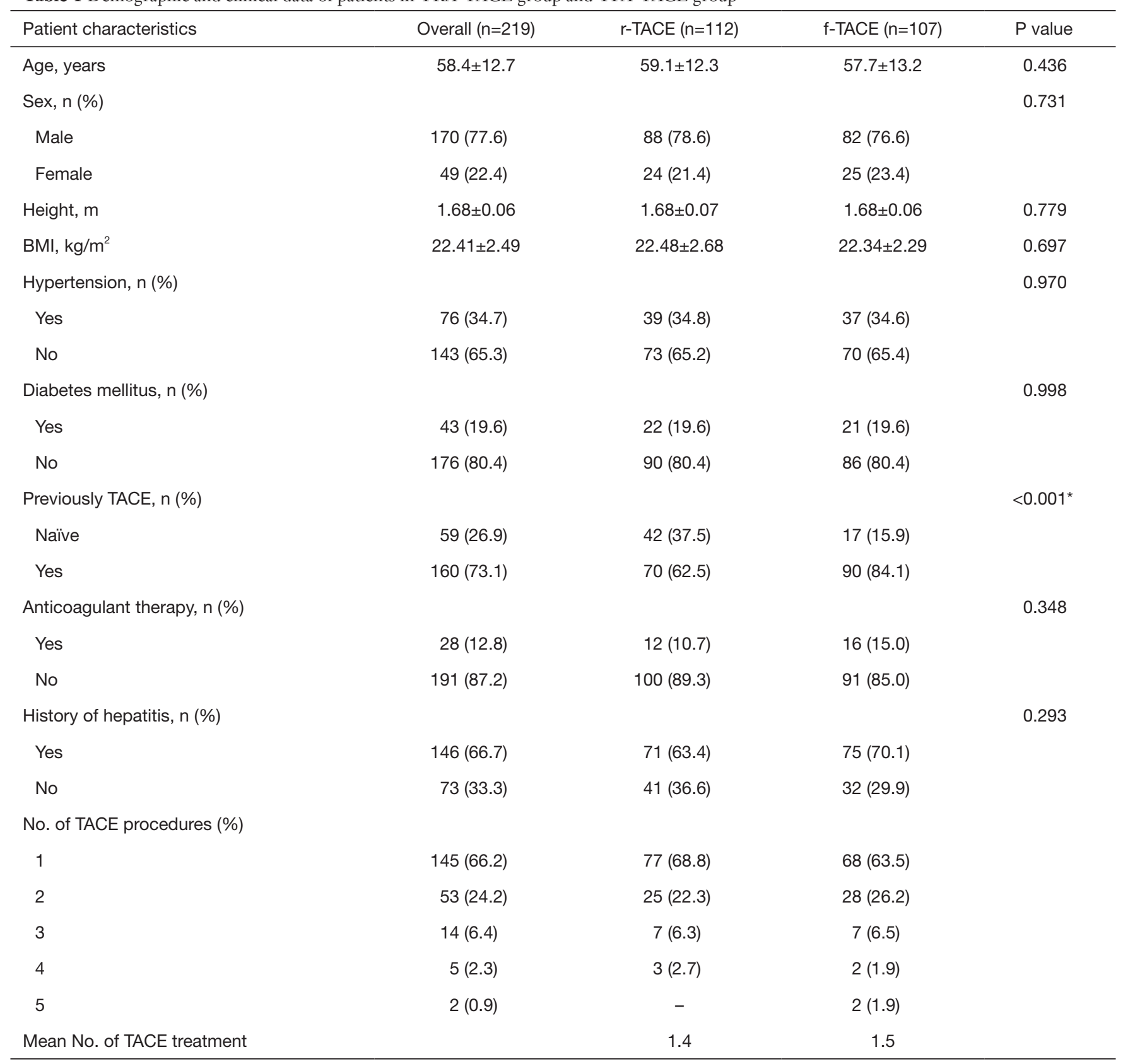

TRA, transradial access; TACE, transarterial chemoembolization; BMI, body mass index; No., number. ${ }^{*} \mathrm{P}<0.05$.

likely to suffer access site-related bleeding complications. Third, repeat TRA is clinically safe and feasible for patients undergoing TACE.

Transradial PCI has been demonstrated as a safe and feasible technique for patients with coronary disease $(20,21)$. In a RIVAL trial, TRA PCI and TFA PCI had similar procedural success rates; however, in TRA PCI, $7.6 \%$ of the cases changed vascular access site, and in TFA PCI the rate was $2.0 \%$ (11). In general, TRA is more technically challenging for IRs than TFA. In our study, three cases (1.9\%) received access site crossover from RA to FA for difficult cannulation. A retrospective study analyzed RA access for noncoronary interventions, indicating that the technical success rate was $98.2 \%(1,485 / 1,512)$ and only 27 cases 
Table 2 Clinical data and outcomes in each TACE procedures between TRA-TACE group and TFA-TACE group

\begin{tabular}{|c|c|c|c|c|}
\hline Characteristics & Overall $(n=323)$ & $r-$ TACE $(n=160)$ & f-TACE $(n=163)$ & $P$ value \\
\hline Class A & 272 (84.2) & $131(81.9)$ & $141(86.5)$ & \\
\hline Class B & $51(15.8)$ & $29(18.1)$ & $22(13.5)$ & \\
\hline INR value, n (\%) & & & & 0.453 \\
\hline$<1.5$ & $304(94.1)$ & $149(93.1)$ & $155(95.1)$ & \\
\hline PT value, n (\%) & & & & 0.922 \\
\hline$>15 \mathrm{~s}$ & $43(13.3)$ & $21(13.1)$ & $22(13.5)$ & \\
\hline$\leq 15 \mathrm{~s}$ & $280(86.7)$ & $139(86.9)$ & $141(86.5)$ & \\
\hline Multiple & $204(63.2)$ & $99(61.9)$ & $105(64.4)$ & \\
\hline Tumor location & & & & 0.155 \\
\hline Left & $49(15.2)$ & $19(11.9)$ & $30(18.4)$ & \\
\hline Right & $157(48.6)$ & $85(53.1)$ & $72(44.2)$ & \\
\hline Bilateral & $117(36.2)$ & $56(35.0)$ & $61(37.4)$ & \\
\hline Technical success, n (\%) & & & & 0.102 \\
\hline Success & $313(96.9)$ & $152(95.0)$ & $161(98.8)$ & \\
\hline Failure & $10(3.1)$ & $8(5.0)$ & $2(1.2)$ & \\
\hline Vascular complications & - & - & - & \\
\hline RAO & - & $3(1.9)$ & - & \\
\hline Bleeding complications & & & & $0.003^{*}$ \\
\hline Minor & - & - & $6(3.7)$ & \\
\hline Major & - & - & $3(1.8)$ & \\
\hline Postoperative dysuresia, n (\%) & & & & $0.030^{*}$ \\
\hline Yes & $6(1.9)$ & - & $6(3.7)$ & \\
\hline No & $317(98.1)$ & $160(100.0)$ & $157(96.3)$ & \\
\hline
\end{tabular}

TRA, transradial access; TFA, transfemoral access; TACE, transarterial chemoembolization; INR, international normalized ratio; PT, prothrombin time; $\mathrm{RAO}$, radial artery occlusion; $\mathrm{PA}$, pseudoaneurysm. ${ }^{*}, \mathrm{P}<0.05$. 
Table 3 Comparison of the cases with or without access site-related bleeding complications in TFA-TACE group

\begin{tabular}{|c|c|c|c|}
\hline $\begin{array}{l}\text { Patient } \\
\text { characteristics }\end{array}$ & $\begin{array}{c}\text { Cases with } \\
\text { complications } \\
\quad(n=9)\end{array}$ & $\begin{array}{l}\text { Cases without } \\
\text { complications } \\
\quad(n=154)\end{array}$ & $P$ value \\
\hline Age, years & $72.6 \pm 7.9$ & $57.1 \pm 12.7$ & $<0.001^{\star}$ \\
\hline Sex, n (\%) & & & 1.000 \\
\hline Male & $7(77.8)$ & $118(76.6)$ & \\
\hline Female & $2(22.2)$ & $36(23.4)$ & \\
\hline Height, m & $1.66 \pm 0.05$ & $1.68 \pm 0.06$ & 0.385 \\
\hline BMI, $\mathrm{kg} / \mathrm{m}^{2}$ & $22.87 \pm 2.20$ & $22.25 \pm 2.59$ & 0.480 \\
\hline \multicolumn{2}{|c|}{ Hypertension, n (\%) } & & 1.000 \\
\hline Yes & $4(44.4)$ & $61(39.6)$ & \\
\hline No & $5(55.6)$ & $93(60.4)$ & \\
\hline \multicolumn{2}{|c|}{ Diabetes mellitus, n (\%) } & & 0.563 \\
\hline Yes & 3 (33.3) & $30(19.5)$ & \\
\hline No & $6(66.7)$ & $124(80.5)$ & \\
\hline \multicolumn{2}{|c|}{ Previously TACE, n (\%) } & & 1.000 \\
\hline Yes & $1(11.1)$ & $18(11.7)$ & \\
\hline No & 8 (88.9) & $136(88.3)$ & \\
\hline \multicolumn{2}{|c|}{ Anticoagulant therapy, n (\%) } & & 1.000 \\
\hline Yes & $2(22.2)$ & $28(18.2)$ & \\
\hline No & $7(77.8)$ & $126(81.8)$ & \\
\hline \multicolumn{2}{|c|}{ Child-Pugh classification, n (\%) } & & 0.775 \\
\hline Class A & $7(77.8)$ & $134(87.0)$ & \\
\hline Class B & $2(22.2)$ & $20(13.0)$ & \\
\hline INR value, $\mathrm{n}(\%)$ & & & 0.064 \\
\hline$\geq 1.5$ & 2 (22.2) & $6(3.9)$ & \\
\hline$<1.5$ & 7 (77.8) & $148(96.1)$ & \\
\hline PT value, n (\%) & & & $0.022^{*}$ \\
\hline$\leq 15 \mathrm{~s}$ & 5 (55.6) & $136(88.3)$ & \\
\hline$>15 \mathrm{~s}$ & $4(44.4)$ & $18(11.7)$ & \\
\hline
\end{tabular}

TFA, transfemoral access; TACE, transarterial chemoembolization; $\mathrm{BMI}$, body mass index; INR, international normalized ratio; PT, prothrombin time. *, $\mathrm{P}<0.05$.

(1.8\%) required crossover to FA access (22). Consistent with previous reports, the success rate of TACE with TRA in our study is acceptably high and similar to that of TACE with TFA (95.0\% vs. $98.8 \%, \mathrm{P}=0.102)$. Compared with $5.5 \%$ of case in the TFA-TACE group, no cases suffered access siterelated bleeding complications in the TRA-TACE group, which indicated that TRA can reduce the rate of access siterelated bleeding complications in contrast with TFA.

In addition to reducing bleeding complications, patients receiving TRA also have reduced overall discomfort and limitations in performing basic activities after TACE procedure. For TFA patients, the mandatory requirement to stay in bed for at least six hours has become the main concern and source of discomfort. Besides, such a mandatory requirement can increase the bleeding risk, as well as the nursing workload. Previous studies have recorded the degree of postoperative discomfort and patients' satisfaction with the procedure used in both TRA and TFA cases using a qualitative evaluation scale, and the results revealed that patients who underwent TRA-TACE were significantly more comfortable and had strong tendency to choose radial access in the future $(23,24)$. For patients who underwent TFA-TACE, the requirement to straighten their legs meant that they were unable to find a comfortable position. When patients suffer from dysuria, frequent nausea or vomiting, extra nursing care or an indwelling catheter may be required. In our study, 3.7\% of cases received indwelling catheters in the TFA-TACE group, which indicates an increased discomfort for patients and a potential increase in nursing care. Several large-scale randomized trials have demonstrated the reduced incidence of acute kidney injury via $\mathrm{RA}$ interventions contrasted by FA approach $(25,26)$. In our study, no patients suffered from contrast medium-related nephropathy or neurological symptoms in either groups.

In this study, five cases of left RA puncture failure were successfully performed TACE procedure via the right RA access (Figure 2). For patients who underwent TRA-TACE, RA puncture in the left hand was the first choice because the guidewire/catheter can easily pass the aortic arch to the abdominal aorta through the left subclavian artery. This was widely welcomed by the majority of patients, because most people were right-handed. Also, their use of the dominant upper limb would not be restricted during the hemostatic compression after sheath removal (27), as is the case for patients with advanced age or obese weight (Figure 3). The rate of RAO after TRA was $2.7 \%$ (3 of 112 patients) in this study, even with some patients receiving TACE via TRA more than once. Moreover, no RAO patients experienced paresthesia, pain at the site of occlusion, hand function loss or distal ischemia. Overall, despite the transitory or persistent RAO, preserved blood supply to the fingers was ensured by the rich anastomotic and collateral network 

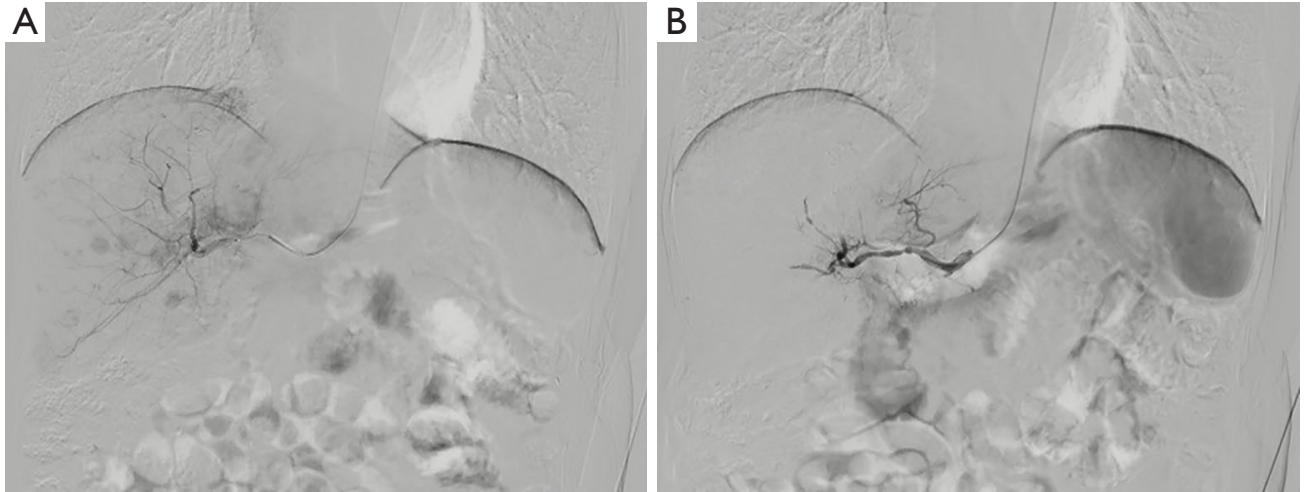

Figure 2 TRA-TACE via right radial artery. (A) The common hepatic arteriogram in a 70-year-old patient shows multiple HCC as hypervascularity lesion in the right lobe of liver; (B) after embolization with iodized oil and gelfoam particles, final angiography shows no flow in the distal part of the artery. TRA, transradial access; TACE, transarterial chemoembolization; HCC, hepatocellular carcinoma.
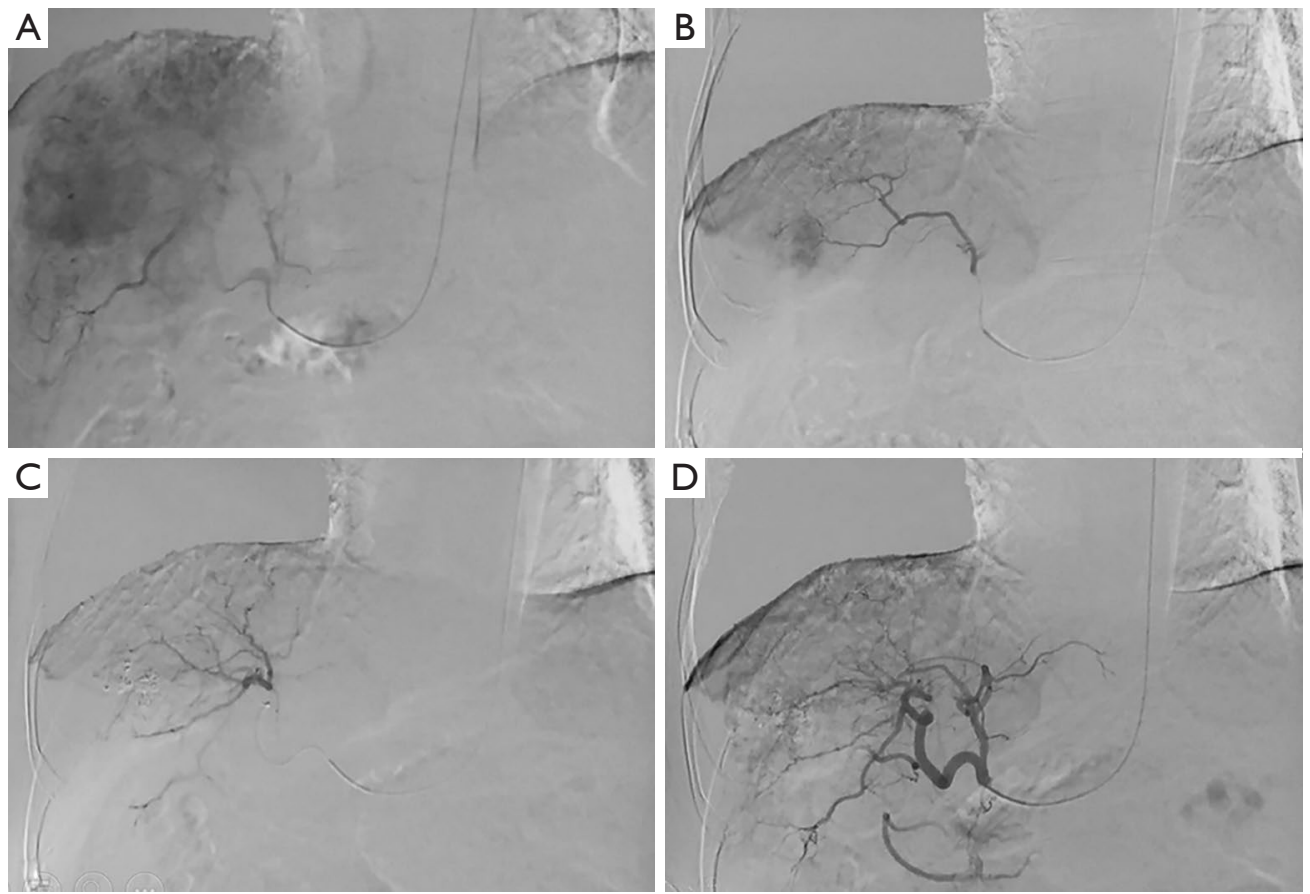

Figure 3 TRA-TACE in severe obese patients (BMI: $41.2 \mathrm{~kg} / \mathrm{m}^{2}$ ) (A) The common hepatic arteriogram shows the hypervascular tumor lesion; (B) super-selective catheterize to the feeding artery via micro-catheter; (C) lipiodol deposition on angiographic imaging after injection of cytotoxic lipiodol emulsion; (D) disappearance of the tumor vascularity and stain on control angiogram after embolization. TRA, transradial access; TACE, transarterial chemoembolization.

between the radial and ulnar arteries (27). Due to the rarity of RAO, we were unable to find a significant correlation between the increased RAO risk and the number of TRA procedures. Yet our study still found the possibility of the progressive RA stenosis with the increased number of
RA punctures. The technical details that may beneficial to reduce the rate of puncture failure and to reduce the occurrence of RAO were summarized as follows: first, a successful first-time RA puncture is important to reduce the number of punctures and to reduce the injury of RA, 
because the distal RA may slip at times. Second, using a small diameter or slender introducer sheath may represent a wise first choice Third, hemostasis should be performed with an incompletely blocked radial pulse, and the Band device should be incrementally deflated once every two hours, with pressure occurring no longer than six hours, which may also help the timely recovery of RA.

As shown in Table 3, patients with advanced age were more likely to suffer access site-related bleeding complications in the TFA-TACE group, as was true for patients with PT $>15$ s. It was noticeable that senile patients could not tolerate long-term bed rest with straightened legs. Also, bleeding risk was more likely to increase for patient with liver dysfunction or anticoagulant therapy. Owing to easy hemostasis, TRA appears to be an attractive selection to reduce access site-related bleeding complications.

Due to the inter-machines variation in fluoroscopic and digital subtraction angiography (DSA) protocols, dose area product (DAP) difference was not assessed or compared between the two groups. Previous literature demonstrated that TRA leads to prolonged fluoroscopy time and increased radiation dose for patients $(28,29)$; however, in the study performed by Jolly et al. (30), over 3,500 patients were randomized to undergo radial or femoral access as part of the RIVAL trial, and their air kerma or the dose-area product were evaluated. Results showed no difference in the dose-area product between the two groups. Previous studies have also shown that increased experience of physicians could be related to the reduced total dose of fluoroscopy exposure (31). In our study, there was no difference in fluoroscopy exposure between the two groups. The potential prolonged fluoroscopy time and higher radiation dose in several cases may attribute to the artery tortuosity, the difficult catheterization from the subclavian artery to the descending aorta, or the difficult catheterization of the celiac artery. With more experience gained and different catheters used by the physician, the difference of fluoroscopy time and radiographic exposure became insignificant between the two access sites used in the TACE procedure. Also, the physician's expertise and experience accumulation are of great importance to improve the technical success rate, as well as the patient's overall safety and comfort.

Although the innovative compression device was not used or compared in the TFA-TACE group, TRA-TACE is still the perfect alternative to reduce related complications, especially for patients with advanced age or PT $>15 \mathrm{~s}$.

\section{Limitations}

The biggest limitation of the current study was the selection bias caused by the retrospective nature. The small sample size also limited the statistical analysis power in the assessment of the technical success rate and the risk factors of access site complications in the TFA-TACE group. Besides, the access route was ultimate decided by the experienced operator, which introduced additional selection bias. Also, the fluoroscopy time and total radiation dose were not recorded and compared between the two groups. Lastly, we lack mid-term and long-term outcome to evaluate the safety and efficacy of r-TACE.

\section{Conclusions}

Transradial TACE is a safe and effective procedure associated with significantly lower rates of access siterelated bleeding and vascular complications in comparison with the femoral approach, without compromising the procedural success rate. It is especially beneficial to patients with advanced age, or those with longer PT. With a low rate of RAO, repeated use of RA puncture is clinically feasible.

\section{Acknowledgments}

We should give our thanks to the departments of medical record and Interventional Radiologist to offer data records. The authors are very grateful to Dr. Xianglin $\mathrm{Hu}$ in Zhongshan Hospital of Fudan University for his very professional suggestions to English writing.

Funding: This work was supported by the Project of Scientific Research from Science and Technology Commission of Shanghai Municipality (16411968600, 17YF1417500).

\section{Footnote}

Conflicts of Interest: All authors have completed the ICMJE uniform disclosure form (available at http://dx.doi. org/10.21037/tcr.2019.08.40). The authors have no conflicts of interest to declare.

Ethical Statement: The authors are accountable for all aspects of the work in ensuring that questions related to the accuracy or integrity of any part of the work are appropriately investigated and resolved. The study was 
conducted in accordance with the Declaration of Helsinki (as revised in 2013). This study was approved by the Ethics Committee of Zhongshan Hospital, Fudan University (Num.2009-080). All patients signed informed consent before operation.

Open Access Statement: This is an Open Access article distributed in accordance with the Creative Commons Attribution-NonCommercial-NoDerivs 4.0 International License (CC BY-NC-ND 4.0), which permits the noncommercial replication and distribution of the article with the strict proviso that no changes or edits are made and the original work is properly cited (including links to both the formal publication through the relevant DOI and the license). See: https://creativecommons.org/licenses/by-nc-nd/4.0/.

\section{References}

1. Iezzi R, Pompili M, Posa A, et al. Transradial versus Transfemoral Access for Hepatic Chemoembolization: Intrapatient Prospective Single-Center Study. J Vasc Interv Radiol 2017;28:1234-9.

2. Hamon M, Pristipino C, Di Mario C, et al. Consensus document on the radial approach in percutaneous cardiovascular interventions: position paper by the European Association of Percutaneous Cardiovascular Interventions and Working Groups on Acute Cardiac Care $^{* *}$ and Thrombosis of the European Society of Cardiology. EuroIntervention 2013;8:1242-51.

3. Jolly SS, Amlani S, Hamon M, et al. Radial versus femoral access for coronary angiography or intervention and the impact on major bleeding and ischemic events: a systematic review and meta-analysis of randomized trials. Am Heart J 2009;157:132-40.

4. Romagnoli E, Biondi-Zoccai G, Sciahbasi A, et al. Radial versus femoral randomized investigation in ST-segment elevation acute coronary syndrome: the RIFLE-STEACS (Radial Versus Femoral Randomized Investigation in STElevation Acute Coronary Syndrome) study. J Am Coll Cardiol 2012;60:2481-9.

5. Vogl TJ, Trapp M, Schroeder H, et al. Transarterial chemoembolization for hepatocellular carcinoma: volumetric and morphologic CT criteria for assessment of prognosis and therapeutic success-results from a liver transplantation center. Radiology 2000;214:349-57.

6. Llovet JM, Bruix J. Systematic review of randomized trials for unresectable hepatocellular carcinoma: Chemoembolization improves survival. Hepatology
2003;37:429-42.

7. Wu T, Sun R, Huang Y, et al. Transradial arterial chemoembolization reduces complications and costs in patients with hepatocellular carcinoma. Indian J Cancer 2015;52:e107-11.

8. Shiozawa S, Tsuchiya A, Endo S, et al. Transradial approach for transcatheter arterial chemoembolization in patients with hepatocellular carcinoma - Comparison with conventional transfemoral approach. J Clin Gastroenterol 2003;37:412-7.

9. Mortensen C, Chung J, Liu D, et al. Prospective Study on Total Fluoroscopic Time in Patients Undergoing Uterine Artery Embolization: Comparing Transradial and Transfemoral Approaches. Cardiovasc Intervent Radiol 2019;42:441-7.

10. Rao SV, Cohen MG, Kandzari DE, et al. The transradial approach to percutaneous coronary intervention: historical perspective, current concepts, and future directions. J Am Coll Cardiol 2010;55:2187-95.

11. Jolly SS, Yusuf S, Cairns J, et al. Radial versus femoral access for coronary angiography and intervention in patients with acute coronary syndromes (RIVAL): a randomised, parallel group, multicentre trial. Lancet 2011;377:1409-20.

12. Bernat I, Horak D, Stasek J, et al. ST-segment elevation myocardial infarction treated by radial or femoral approach in a multicenter randomized clinical trial: the STEMIRADIAL trial. J Am Coll Cardiol 2014;63:964-72.

13. Uhlemann M, Mobius-Winkler S, Mende M, et al. The Leipzig Prospective Vascular Ultrasound Registry in Radial Artery Catheterization Impact of Sheath Size on Vascular Complications. JACC Cardiovasc Interv 2012;5:36-43.

14. Shemesh D, Goldin I, Verstandig A, et al. Upper limb grafts for hemodialysis access. J Vasc Access 2015;16 Suppl 9:S34-9.

15. Head SJ, Milojevic M, Taggart DP, et al. Current Practice of State-of-the-Art Surgical Coronary Revascularization. Circulation 2017;136:1331-45.

16. Masden DL, McClinton MA. Arterial conduits for distal upper extremity bypass. J Hand Surg Am 2013;38:572-7.

17. Zhu AX, Baron $\mathrm{AD}$, Malfertheiner $\mathrm{P}$, et al. Ramucirumab as Second-Line Treatment in Patients With Advanced Hepatocellular Carcinoma Analysis of REACH Trial Results by Child-Pugh Score. JAMA Oncol 2017;3:235-43.

18. Dariushnia SR, Gill AE, Martin LG, et al. Quality improvement guidelines for diagnostic arteriography. J Vasc Interv Radiol 2014;25:1873-81.

19. Patel IJ, Davidson JC, Nikolic B, et al. Consensus 
guidelines for periprocedural management of coagulation status and hemostasis risk in percutaneous image-guided interventions. J Vasc Interv Radiol 2012;23:727-36.

20. Feldman DN, Swaminathan RV, Kaltenbach LA, et al. Adoption of radial access and comparison of outcomes to femoral access in percutaneous coronary intervention: an updated report from the national cardiovascular data registry (2007-2012). Circulation 2013;127:2295-306.

21. Tanaka Y, Moriyama N, Ochiai T, et al. Transradial Coronary Interventions for Complex Chronic Total Occlusions. JACC Cardiovasc Interv 2017;10:235-43.

22. Posham R, Biederman DM, Patel RS, et al. Transradial Approach for Noncoronary Interventions: A Single-Center Review of Safety and Feasibility in the First 1,500 Cases. J Vasc Interv Radiol 2016;27:159-66.

23. Yamada R, Bracewell S, Bassaco B, et al. Transradial Versus Transfemoral Arterial Access in Liver Cancer Embolization: Randomized Trial to Assess Patient Satisfaction. J Vasc Interv Radiol 2018;29:38-43.

24. Yuan DZ, Brooks M, Dabin B, et al. Radial versus femoral access for cardiac catheterisation: Impact on quality of life. Int J Cardiol 2015;178:91-2.

25. Andò G, Cortese B, Russo F, et al. Acute Kidney Injury After Radial or Femoral Access for Invasive Acute Coronary Syndrome Management: AKI-MATRIX. J Am

Cite this article as: Du N, Yang MJ, Ma JQ, Luo JJ, Zhang ZH, Yu TZ, Zheng ZY, Zhang W, Yan ZP. Transradial access chemoembolization for hepatocellular carcinoma in comparation with transfemoral access. Transl Cancer Res 2019;8(5):1795-1805. doi: 10.21037/tcr.2019.08.40
Coll Cardiol 2017. [Epub ahead of print].

26. Valgimigli M, Gagnor A, Calabro P, et al. Radial versus femoral access in patients with acute coronary syndromes undergoing invasive management: a randomised multicentre trial. Lancet 2015;385:2465-76.

27. Sgueglia GA, Di Giorgio A, Gaspardone A, et al. Anatomic Basis and Physiological Rationale of Distal Radial Artery Access for Percutaneous Coronary and Endovascular Procedures. JACC Cardiovasc Interv 2018;11:2113-9.

28. Kis B, Mills M, Hoffe SE. Hepatic radioembolization from transradial access: initial experience and comparison to transfemoral access. Diagn Interv Radiol 2016;22:444-9.

29. Neill J, Douglas H, Richardson G, et al. Comparison of radiation dose and the effect of operator experience in femoral and radial arterial access for coronary procedures. Am J Cardiol 2010;106:936-40.

30. Jolly SS, Cairns J, Niemela K, et al. Effect of radial versus femoral access on radiation dose and the importance of procedural volume: a substudy of the multicenter randomized RIVAL trial. JACC Cardiovasc Interv 2013;6:258-66.

31. Lo TS, Ratib K, Chong AY, et al. Impact of access site selection and operator expertise on radiation exposure; a controlled prospective study. Am Heart J 2012;164:455-61. 